\title{
Post-traumatic thrombo-embolic complications in polytrauma patients
}

\author{
Philipp Lichte • Philipp Kobbe • Khalid Almahmoud • Roman Pfeifer • \\ Hagen Andruszkow • Frank Hildebrand • Rolf Lefering • Hans-Christoph Pape • \\ Trauma Register DGU
}

Received: 15 January 2015 / Accepted: 27 January 2015 / Published online: 18 February 2015

(C) SICOT aisbl 2015

\begin{abstract}
Introduction Thrombo-embolic events after trauma are considered to be life-threatening complications. Our aim was to determine the incidence of arterial and venous thromboembolic events (TE) in severely-injured trauma patients, and its associated risk factors by using a large trauma registry.
\end{abstract}

\footnotetext{
P. Lichte $(\bowtie) \cdot$ P. Kobbe $\cdot$ K. Almahmoud $\cdot$ R. Pfeifer $\cdot$

H. Andruszkow $\cdot$ F. Hildebrand $\cdot$ H.-C. Pape

Department of Orthopaedic Trauma Surgery, University Hospital

Aachen, Pauwelsstraße 30, 52074 Aachen, Germany

e-mail: plichte@ukaachen.de

P. Kobbe

e-mail: pkobbe@ukaachen.de

K. Almahmoud

e-mail:kalmahmoud@ukaachen.de

R. Pfeifer

e-mail: rpfeifer@ukaachen.de

H. Andruszkow

e-mail: handruszkow@ukaachen.de

F. Hildebrand

e-mail: fhildebrand@ukaachen.de

H.-C. Pape

e-mail: hpape@ukaachen.de

K. Almahmoud

Department of Surgery, University of Pittsburgh,

Pittsburgh, PA 15213, USA

\section{R. Lefering}

Institute for Research in Operative Medicine (IFOM), University of Witten/Herdecke, Ostmerheimer Str. 200, 51109 Cologne, Germany

e-mail: Rolf.Lefering@uni-wh.de

R. Pfeifer $\cdot$ H. Andruszkow

Harald Tscherne Research Laboratory for Orthopaedic Trauma, Department of Orthopaedic Trauma Surgery, University Hospital RWTH Aachen, Aachen, Germany
}

Methods Patients' data from the TraumaRegister DGU ${ }^{\circledR}$ (TR-DGU) were screened for TE (DVT [symptomatic deep vein thrombosis], PE [symptomatic pulmonary embolism], MI [myocardial infarction], and stroke) through the clinical course of severely injured adult trauma patients from January 2005 to December 2012. Univariate analysis was used to compare the clinical outcomes (endpoints: mortality, ICU and hospital length of stay, ventilator days), and a multivariate regression analysis was used to assess the independent risk factors associated with each TE event.

Results From a cohort of 40,846 trauma patients, 1122 (2.8\%) patients developed a TE during their posttraumatic clinical course $(313,0.8 \%$ DVT; 425, $1.0 \% \mathrm{PE} ; 160,0.4 \% \mathrm{MI}$ and 231, $0.6 \%$ stroke). ICU length of stay [LOS], total LOS, days on mechanical ventilation, and incidence of multiple organ failure (MOF) and sepsis were significantly increased in patients with TE complications. Injury severity, major pelvic injury, and one or more operations were found to be independent risk factors for the development of DVT. Age $\geq 60$ years, male gender, and more than one operation were risk factors for PE development. For MI age was the only significant risk factor. The occurrence of a stroke is increased in patients with an age $\geq 60$ years, major head injury (AIS head $\geq 3$ ), and more than one operation. Finally, mortality rates were significantly higher in the TE group when compared to the non-TE cohort $(21.8 \%$ vs. $12.7 \% ; p<0.001)$.

Conclusion TE complications were associated with longer ICU and hospital stay as well as a higher mortality. Overall, age and repeated operations were the most important risk factors for the development of TE events.

Keywords Polytrauma $\cdot$ Embolic complication $\cdot$ Risk factor 


\section{Introduction}

Thrombo-embolic complications after trauma are considered to represent significant contributors for morbidity and mortality [1]. Despite the widespread use of prophylactic protocols, the incidence of DVT and PE is still high during the clinical course post-injury [2]. Within the discipline of trauma and orthopaedics, thrombo-embolic complications are the most common preventable cause of in-hospital deaths [2]. Several risk factors for developing thrombo-embolic complication have been identified: age, long bone and pelvic fractures, spinal cord and traumatic brain injuries, prolonged immobilization, and delay of prophylactic management [3].

Thrombo-embolic complications in the post-injury phase are venous and arterial. Several studies have explored the venous complications but very few focused on the arterial TE. Previous studies showed that venous and arterial thrombo-embolic complications share a number of risk factors in non-trauma patients [4]; however, trauma-specific risk factors for development of arterial thrombo-embolic events in severely injured trauma patients are not yet fully elucidated. Recent studies implicate traumatic brain injury as a risk factor for ischemic stroke post-injury [5], and peri-operative haemorrhage for stroke and myocardial infarction [6].

Trauma patients differ based on type of injury, location, and additional factors such as the individual's sex, the length of the interval between injury and surgery, the presence of shock at time of presentation, and the presence of cranial injuries [7]. The identification of individual risk factors is proven to be crucial for individual prophylaxis and therapy [8, 9], which has been recently applied in practice through personalized clinical management of thrombosis $[4,10]$.

Aims of this study were:

- To determine the incidence of different thrombo-embolic complications, e.g. symptomatic deep venous thrombosis (DVT), symptomatic pulmonary embolism (PE), myocardial infarction (MI), and stroke in severely injured trauma patients, and to compare the outcome of patients with and without thrombo-embolic complications.

- To identify the associated risk factors for various thrombo-embolic complications in a nationwide registry.

\section{Materials and methods}

Patients

Data were obtained by the TraumaRegister DGU ${ }^{\circledR}$ (TR-DGU, German Trauma Society; Deutsche Gesellschaft für Unfallchirurgie [DGU]). Patients documented between January 1 st 2005 and December 31st 2012 were eligible. We excluded children (age $<16$ years) and patients transferred out within 48 hours. Patients with an Injury Severity Score (ISS) of less than 9 points were excluded.

The TraumaRegister DGU ${ }^{\circledR}$ of the German Trauma Society (Deutsche Gesellschaft für Unfallchirurgie, DGU) was founded in 1993. The aim of this multi-centre database is an anonymous and standardized documentation of severely injured patients. Data are collected prospectively in four consecutive time phases from the site of the accident until discharge from hospital: (1) pre-hospital phase, (2) emergency room and initial surgery, (3) intensive care unit and (4) discharge. The documentation includes detailed information on demographics, injury pattern, comorbidities, pre- and in-hospital management, course on intensive care unit, as well as relevant laboratory findings including data on transfusion and outcome of each individual. The inclusion criterion is admission to hospital via emergency room with subsequent ICU/ICM care or reaching the hospital with vital signs and dying before admission to ICU.

The infrastructure for documentation, data management, and data analysis was provided by AUC-Academy for Trauma Surgery (AUC-Akademie der Unfallchirurgie GmbH), a company affiliated with the German Trauma Society. The scientific leadership is provided by the Committee on Emergency Medicine, Intensive Care and Trauma Management (Sektion NIS) of the German Trauma Society. The participating hospitals submit their data anonymously into a central database via a web-based application. Scientific data analysis is approved according to a peer review procedure established by Sektion NIS.

The participating hospitals are primarily located in Germany $(90 \%)$, but a rising number of hospitals of other countries contribute data as well (at the moment from Austria, Belgium, China, Finland, Luxembourg, Slovenia, Switzerland, The Netherlands, and the United Arab Emirates). Currently, approximately 25,000 cases from more than 600 hospitals are entered into the database per year.

Participation in TraumaRegister DGU ${ }^{\circledR}$ is voluntary. For hospitals associated with TraumaNetzwerk DGU ${ }^{\circledR}$, however, the entry of at least a basic data set is obligatory for reasons of quality assurance and operative procedures. Data are submitted to a central web-based database that is hosted by AUC (Akademie der Unfallchirurgie $\mathrm{GmbH}$ ) of the DGU (www. traumaregister.de).

The present study is in line with the publication guidelines of the TraumaRegister DGU ${ }^{\circledR}$ and registered as TR-DGU project ID 2013-054.

\section{Clinical data}

Clinical data, including age, gender, Injury Severity Score (ISS), ICU length of stay (LOS), hospital LOS, days on mechanical ventilator, and mortality rates were collected from the 
TR-DGU. All injuries were coded according to the Abbreviated Injury Scale (AIS, version 2005) and the ISS was calculated from these codes. The ISS is an anatomical scoring system that provides an overall score for patients with severe injuries. Each injury is assigned an AIS severity score, allocated to one of six body regions: head/neck, face, chest, abdomen, extremities (including pelvis), and external. Only the highest AIS score in each body region is used. To calculate the ISS, the scores of the three most severely injured body regions are squared and added to give the ISS score.

\section{Definitions and endpoints}

\section{Thrombo-embolic events}

Thrombo-embolic events (symptomatic deep venous thrombosis (DVT), symptomatic pulmonary embolism (PE), myocardial infarction (MI), and stroke) were documented by the treating physician. All patients without documented TE were included in the non-TE group. Use of prophylactic management was reported briefly as "yes/no" in case an event was observed.

\section{Coagulopathy and treatment}

Coagulopathy was defined as prothrombin time (PT) test $<$ $70 \%$ of normal activity (Quick's value), activated partial thromboplastin time $(\mathrm{aPTT})>40 \mathrm{sec}$, or platelet counts $<100$, $000 / \mu 1$.

Shock at admission was defined as systolic blood pressure $(\mathrm{SBP})<90 \mathrm{~mm} / \mathrm{Hg}$. Haemostasis therapy through the first 48 hours was documented by treating physician and included the application of fibrinogen, rFVIIa, prothrombin complex concentrate, and fibrinolytic inhibitors. Furthermore, the number of transfused packed red blood cell (PRBCs) units was reported.

\section{Complications}

Several complications during the clinical course were assessed. The diagnosis of sepsis was made according to the criteria of the American College of Chest Physicians/ Society of Critical Care Medicine (ACCP/SCCM) consensus conference committee. Organ function status was evaluated according to the Sequential Organ Failure Assessment (SOFA) score. With 3 or more points, an organ function was considered as failure, while multiple organ failure (MOF) was defined as simultaneous failure of at least two organ systems.
Statistical analysis

The statistical analysis was performed using Statistical Package for Social Sciences (SPSS) (IBM Inc., Armonk, NY, USA). Continuous variables are reported as mean and standard deviation (SD), and incidence rates as percentages. Multivariate logistic regression analyses were performed to assess the risk factors associated with each TE event (PE, DVT, MI, and stroke) as an dependent variable. The following variables were considered as potential predictors for TEs: age, gender, ISS, AIS head $\geq 3$, AIS thorax $\geq 3$, AIS abdomen $\geq 3$, AIS pelvis $\geq 2$, number of operations performed (none, one, more than 1), initial coagulopathy, shock on admission, hemostasis therapy, and blood transfusion. Complete documentation of these variables was mandatory for inclusion in the subgroup for multivariate logistic regression analyses. Formal statistical testing was largely avoided due to the large number of variables considered, and the multiplicity of testing if five subgroups are involved. Furthermore, the large sample size ( $>1000$ with TE, $>39,000$ in the control group) would result in highly significant results even for minor and irrelevant differences. Therefore, only selected aspects were tested (U-test for continuous variables, chi-squared test for counts). A pvalue $<0.05$ was considered statistically significant.

\section{Results}

Demographics and characteristics of trauma patients

The overall study cohort consisted of 40,846 trauma patients who met the inclusion criteria. Among them, 1122 (2.8\%) patients developed a documented thromboembolic event during their clinical post-traumatic course. Of these, 313 (28.6\%) developed a symptomatic deep vein thrombosis (DVT), 425 (35.7\%) suffered a symptomatic pulmonary embolism (PE), $160(14.3 \%)$ had a myocardial infarction (MI), and 231 (21.4\%) had a stroke. Table 1 provides an overview of characteristics of patients with and without TE. Blunt trauma was predominant in both TE and non-TE cohorts $(96.1 \%$ and $95.3 \%$, respectively) as well as male gender (74\% and $73 \%$, respectively). Age was significantly higher in the TE cohorts when compared to non-TE cohort, with exception for the DVT subgroup. ISS was statistically significantly higher in the TE cohorts when compared to the non-TE cohort (Table 1).

Comparison of injuries by body region

We next compared the AIS in the TE and non-TE cohorts, in order to determine if specific body region injuries are associated with worse outcomes post-trauma in the sub-cohorts. Our analysis didn't show any relevant differences in head/neck, 
Table 1 Demographic data, and clinical characteristics of general thrombo-embolic and non-thrombo-embolic cohorts (thrombo-embolic cohort $n=$ 1,129 ; non-thrombo-embolic cohort $n=39,717$ )

\begin{tabular}{|c|c|c|c|c|c|c|}
\hline Characteristic & DVT & $\mathrm{PE}$ & MI & Stroke & $\begin{array}{l}\text { Thrombo-embolic } \\
\text { event (TE) }\end{array}$ & $\begin{array}{l}\text { Non-thrombo-embolic } \\
\text { event (non-TE) }\end{array}$ \\
\hline No. of cases & 313 & 425 & 160 & 231 & 1,129 & 39,717 \\
\hline Age & 47.7 & 54.1 & 66.8 & 58.5 & 53.8 & 48.0 \\
\hline Male (\%) & 75.8 & 72.8 & 75 & 64.1 & 74.0 & 72.8 \\
\hline Blunt trauma (\%) & 96.4 & 96.6 & 96.8 & 95.1 & 96.1 & 95.3 \\
\hline ISS & 28.4 & 26.9 & 24.1 & 28.8 & 27.7 & 23.7 \\
\hline AIS head $\geq 3(\%)$ & 44.1 & 44.0 & 52.1 & 76.7 & 53.2 & 47.9 \\
\hline AIS chest $\geq 3(\%)$ & 56.5 & 56.5 & 54.6 & 45.7 & 53.8 & 47.7 \\
\hline AIS abdomen $\geq 3(\%)$ & 24.6 & 23.3 & 16.0 & 12.9 & 20.3 & 14.9 \\
\hline AIS extremity $\geq 3(\%)$ & 47.9 & 41.4 & 25.8 & 29.7 & 29.3 & 32.1 \\
\hline AIS pelvis $\geq 2(\%)$ & 31.9 & 25.9 & 19.6 & 19.8 & 25.1 & 19.4 \\
\hline Operation (\%) & 93.3 & 88.3 & 68.9 & 84.2 & 86.6 & 78.4 \\
\hline Blood transfusion (\%) & 24.6 & 25.4 & 13.5 & 21.1 & 24.7 & 17.4 \\
\hline Mass transfusion ( $\geq 10$ PRBCs) $(\%)$ & 5.4 & 5.6 & 2.5 & 5.2 & 5.5 & 3.7 \\
\hline Shock on admission (\%) & 13.2 & 16.1 & 13.7 & 15.5 & 15.8 & 11.3 \\
\hline
\end{tabular}

DVT deep vein thrombosis, PE pulmonary embolism, MI myocardial infarction, ISS Injury Severity Score, AIS Abbreviated Injury Scale,

chest, and abdomen regions between the TE and non-TE groups. However, we observed a significantly higher rate of injuries in the extremities and especially pelvic body region of patients who sustained a venous TE (DVT and PE) when compared to non-TE patients (Table 1).

\section{Clinical outcomes and in-hospital complications}

Our data showed that the overall TE cohort had a statistically significantly higher ICU LOS $(P<0.001)$, hospital LOS $(P<0.001)$, and days on mechanical ventilator $(P<0.001)$ when compared to the non-TE cohort. Moreover, we observed a higher prevalence of sepsis and MOF in TE cohorts when compared to the non-TE cohort. Finally, mortality rates were shown to be significantly higher in the PE, MI, and stroke sub- cohorts when compared to the non-TE, while the DVT group showed a significant lower mortality rate (Table 2).

\section{TE correlate significantly with different parameters}

Multiple factors, including magnitude of injury [11] and age [12], can affect the outcomes of trauma. In this context, we examined the impact of injury severity and age on the rates of TE. Interestingly, our analysis showed a significant increase in the rates of PE, DVT, and stroke with increasing of injury severity while the rate of MI appeared to be unaffected by injury severity (Fig. 1a). Furthermore, we observed a significant increase in rates of MI and stroke with increasing age, while DVT and PE showed a significant decrease in the elderly (Fig. 1b).

Table 2 Clinical outcomes and in-hospital complication of general thrombo-embolic and non-thrombo-embolic cohorts (thrombo-embolic cohort $n=$ 1,129; non-thrombo-embolic cohort $n=39,717$ )

\begin{tabular}{lllllll}
\hline Outcomes and complications & DVT & PE & MI & Stroke & $\begin{array}{l}\text { Thrombo-embolic } \\
\text { event (TE) }\end{array}$ & $\begin{array}{l}\text { Non-thrombo-embolic } \\
\text { event (non-TE) }\end{array}$ \\
\hline Hospital mortality (\%) & $3.2^{*}$ & $27.1^{*}$ & $33.7^{*}$ & $29.7^{*}$ & $21.8^{*}$ & 12.7 \\
Sepsis (\%) & 23.4 & 20.5 & 20.8 & 19.1 & $22.1^{*}$ & 12.9 \\
MOF (\%) & 42.7 & 51.0 & 56.1 & 61.3 & $53.4^{*}$ & 25.7 \\
ICU LOS (days) & $20.4 \pm 19.2$ & $18.6 \pm 18.6$ & $16.3 \pm 18.6$ & $20.0 \pm 17.0$ & $19.6 \pm 19.4^{*}$ & $9.0 \pm 11.9$ \\
Mechanical ventilation (days) & $13.2 \pm 16.5$ & $12.5 \pm 16.4$ & $11.1 \pm 15.2$ & $14.0 \pm 14.1$ & $13.4 \pm 16.7^{*}$ & $5.1 \pm 9.5$ \\
Hospital LOS (days) & $52.4 \pm 37.8$ & $36.9 \pm 27.8$ & $29.6 \pm 27.5$ & $30.4 \pm 26.6$ & $37.8 \pm 31.4^{*}$ & $22.2 \pm 22.6$ \\
\hline
\end{tabular}

$D V T$ deep vein thrombosis, $P E$ pulmonary embolism, $M I$ myocardial infarction, $L O S$ length of stay

Results are presented as means \pm SD. $* P<0.001$ vs. non-TE 

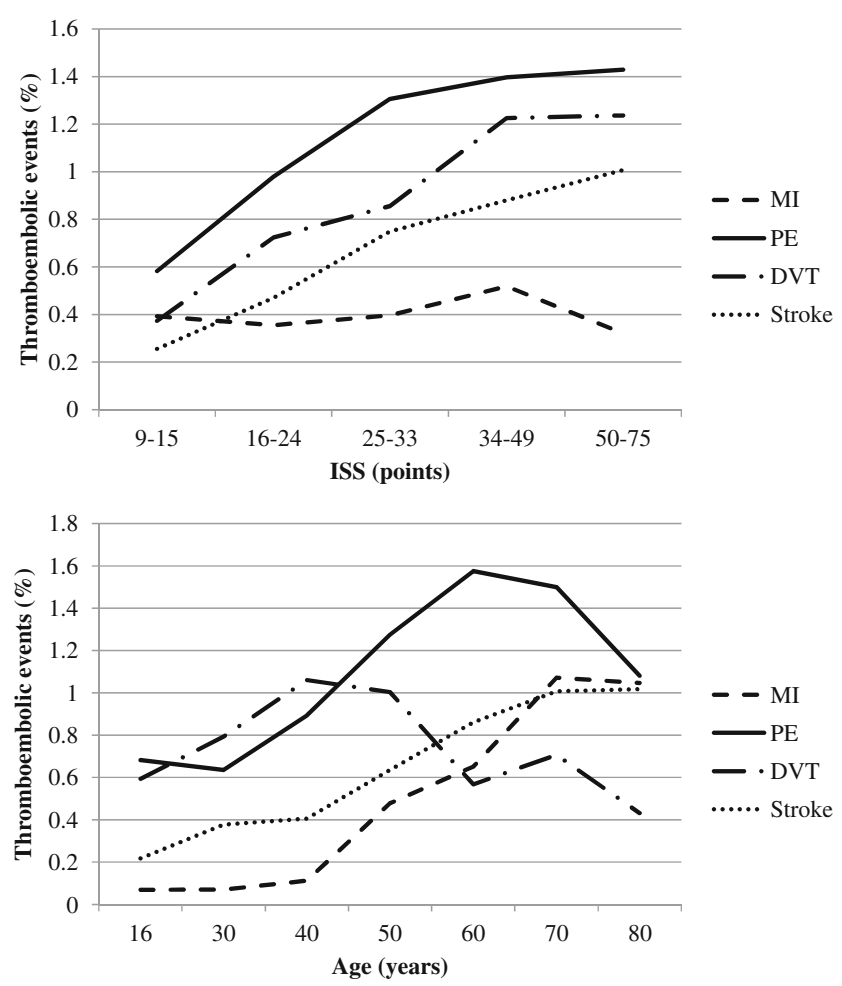

Fig. 1 Impact of injury severity (ISS) (a) and age (b) on the incidence rates of thrombo-embolic events

Risk factors associated with TE

To identify risk factors associated with different TE events by multivariate regression analysis we used a subgroup which is characterized in Table 3. We were able to identify several risk factors associated with different TE events (Table 4). Our analysis showed that the magnitude of injury as reflected by the ISS score, existence of major pelvic injury, and need for one or more operations were independent risk factors for development of DVT. Furthermore, age $\geq 60$ years, male sex, and the need for more than one operation were identified as risk factors for PE. In case of MI, age $\geq 60$ years was the most important factor. Finally, stroke development showed to be dependent on age, major head injury (AIS head $\geq 3$ ), and the need for more than one operation.

\section{Discussion}

During the past decades, improvements in the management of trauma patients through rapid transport to trauma centres, early resuscitation, and the use of damage control approaches have shifted the outcomes from mortality to morbidity which are associated with prolonged ICU and in-hospital LOS, MOF, sepsis, and thrombo-embolic complications.

The main results of the present study were as follows:

- The overall incidence of thrombo-embolic events was $2.8 \%$.

- Pulmonary embolism was diagnosed most frequently (35.7\%), followed by DVT (28.6 \%), stroke (21.4\%), and MI (14.3\%).

- Several independent risk factors for the different entities of TE complications were identified; age $>60$ years (OR 1.98 ) and more than one operation were the most important risk factors (OR 2.04).

- ISS was only an independent risk factor for DVT but not for PE or arterial thrombo-embolism.

Table 3 Characterisation of the selected cohorts for the multivariate regression analysis

\begin{tabular}{|c|c|c|c|c|c|c|}
\hline Characteristic & DVT & $\mathrm{PE}$ & MI & Stroke & $\begin{array}{l}\text { Thrombo-embolic } \\
\text { event (TE) }\end{array}$ & $\begin{array}{l}\text { Non-thrombo-embolic } \\
\text { event (non-TE) }\end{array}$ \\
\hline No. of cases & 182 & 269 & 104 & 150 & 833 & 26194 \\
\hline Male $(\%)$ & 78.6 & 81.8 & 75.0 & 63.3 & 74.5 & 73.2 \\
\hline Age $\geq 60(\%)$ & 25.8 & 41.6 & 67.3 & 52.7 & 41.3 & 28.8 \\
\hline ISS & 27.6 & 26.5 & 24.2 & 28.4 & 27.2 & 23.3 \\
\hline AIS head $\geq 3(\%)$ & 37.4 & 39.8 & 46.2 & 74.0 & 49.9 & 45.9 \\
\hline AIS thorax $\geq 3(\%)$ & 55.5 & 55.0 & 54.8 & 44.7 & 53.4 & 48.6 \\
\hline AIS abdomen $\geq 3(\%)$ & 25.3 & 24.2 & 19.2 & 14.7 & 20.4 & 14.9 \\
\hline AIS pelvis $\geq 2(\%)$ & 35.2 & 26.8 & 23.1 & 21.3 & 26.1 & 19.6 \\
\hline One operation $(\%)$ & 13.2 & 15.6 & 26.9 & 17.3 & 15.8 & 20 \\
\hline More than one operation (\%) & 82.4 & 72.9 & 45.2 & 66.7 & 71.8 & 59.0 \\
\hline Coagulopathy (\%) & 30.2 & 32.7 & 29.8 & 34.0 & 33.9 & 26.4 \\
\hline Blood transfusion (\%) & 28.0 & 29.4 & 14.4 & 22.7 & 27.5 & 19.4 \\
\hline Shock on admission $(\%)$ & 13.7 & 17.8 & 13.5 & 15.3 & 16.4 & 11.1 \\
\hline Haemostasis therapy $(\%)$ & 18.1 & 18.6 & 14.4 & 14.7 & 19.6 & 10.8 \\
\hline
\end{tabular}

$D V T$ deep vein thrombosis, $P E$ pulmonary embolism, $M I$ myocardial infarction, ISS Injury Severity Score, AIS Abbreviated Injury Scale 
Table 4 Independent risk factors for development of DVT, PE, MI, stroke, or any thrombo-embolic event from a multivariate logistic regression analysis

\begin{tabular}{|c|c|c|c|c|c|}
\hline Risk factor & DVT (OR) & PE (OR) & MI (OR) & Stroke (OR) & Any TE (OR) \\
\hline Male sex & n.s. & $1.85^{* * *}$ & n.s. & n.s. & $1.19 *$ \\
\hline Age $\geq 60$ years & n.s. & $2.23 * * *$ & $5.35^{* * *}$ & $2.50 * * *$ & $1.98 * * *$ \\
\hline ISS (per point) & $1.021 * *$ & n.s. & n.s. & n.s. & $1.012 * *$ \\
\hline Head injury (AIS $\geq 3$ ) & $0.68^{*}$ & $0.71 *$ & n.s. & $2.85 * * *$ & n.s. \\
\hline Thoracic injury(AIS $\geq 3$ ) & n.s. & n.s. & n.s. & n.s. & n.s. \\
\hline Abdominal injury (AIS $\geq 3$ ) & n.s. & n.s. & $1.95^{*}$ & n.s. & n.s. \\
\hline Pelvic injury (AIS $\geq 2$ ) & $1.61 * *$ & n.s. & n.s. & n.s. & n.s. \\
\hline One operation & $2.94 * *$ & n.s. & n.s. & n.s. & $1.38^{*}$ \\
\hline More than one operation & $5.18^{* * *}$ & $1.94 * *$ & n.s. & $2.12 * *$ & $2.04 * * *$ \\
\hline Coagulopathy & n.s. & n.s. & n.s. & n.s. & n.s. \\
\hline Blood transfusion & n.s. & n.s. & $0.48^{*}$ & n.s. & n.s. \\
\hline Shock on admission & n.s. & n.s. & n.s. & n.s. & n.s. \\
\hline Haemostasis therapy & n.s. & n.s. & n.s. & n.s. & $1.47 * *$ \\
\hline
\end{tabular}

DVT deep vein thrombosis, $P E$ pulmonary embolism, MI myocardial infarction, ISS Injury Severity Score, AIS Abbreviated Injury Scale, n.s. not significant

The odds ratio (OR) is presented for significant results. ${ }^{*} P<0.05 ; * * P<0.01 ; * * * 0.001$

Venous TE incidence in our study population is in line with previous published results in severely injured trauma patients $[3,13]$ as well as the distribution of PE and DVT $[13,14]$. Only isolated studies reported about lower incidence rates. In contrast to the venous TE, the incidence of post-traumatic arterial TE isn't fully elucidated. Accordingly, our data showed that arterial TE complications were responsible for approximately one third of the post-traumatic TE events, with stroke being more common than MI, which urge the need for specific prophylaxis strategies. Furthermore, arterial TE complications (MI and stroke) are associated with higher mortality, longer hospital and ICU stay, and severe complications such as sepsis and MOF. Previous studies reported similar results for post-traumatic stroke $[5,15]$, whereas data regarding MI in trauma patients is scarce. However, our data is not sufficient to clarify a causative effect for the TE event itself. In this context, the higher overall injury severity in the TE groups has to be considered.

Several factors associated with TE development in the post-injury clinical course have been described $[5,16,17]$; however, most of those studies focused on venous complications (DVT and PE), while arterial TE (MI and stroke) risk factors weren't fully clarified [3, 8, 17-19]. In our trauma patients set, male gender was the only risk factor for PE and overall TE events, which is in agreement with previous studies that showed a weak gender impact on the development of TE complications [5]. Furthermore, age has been described as a significant factor for development of venous TE complications [20]. This has been associated with the increased incidence of comorbidities (e.g. congestive heart failure, malignancy, varicose veins) which predisposes patients to thrombosis [20]. Our analysis showed that age is a risk factor for PE but not for DVT development.

The ISS correlates linearly with outcomes in the posttraumatic clinical course, as well as with the late complications. Our analysis showed that ISS is the main predictive factor for venous thrombo-embolic complications [3, 13]. In addition, DVT rates showed to be affected by pelvic injuries and number of operations needed; however, pelvic injury was identified as an independent risk factor for development of DVT [21]. Furthermore, previous studies identified lower leg injuries and the length of immobilisation as relevant risk factors for the development of DVT [22], while other studies $[14,23]$ didn't find such correlations. This discrepancy to our results might be due to different classifications of pelvic injuries. Additionally, it is well known that number of operative interventions has a relevant impact on the risk to develop TE events after trauma $[3,19,23]$. In our study we identified the presence of more than one operation as an independent risk factor for DVT and PE.

This study was the first to identify the independent risk factors for development of arterial thrombo-embolic complications in the clinical course post-injury. Age showed to be a significant risk factor for developing MI and stroke in a large homogeneous group of severely injured patients. Our results are in line with other studies with heterogeneous study populations of non-surgical populations and surgical patients. In accordance with our study, age was identified as a crucial risk factor for MI, and cerebral infarction development in nontraumatic settings [16]. However, patients with traumatic brain 
injury (TBI) are at higher risk to develop a stroke during their hospital stay without correlation to age [5]. Similar to venous TE, an increased number of operations was an independent risk factor for the development of stroke but not for MI. Moreover, pelvic injuries were found to be less important in developing arterial TE when compared to venous TE.

Severity of traumatic bleeding was measured by shock on admission as well as the amount of PRBCs received to stabilize the homeostatic conditions. Interestingly, our results didn't show a significant effect for shock or blood transfusion in developing stroke or MI. In regards to MI, we observed a protective influence of RBC transfusion. This suggests that a liberal transfusion regime in patients with preexisting coronary disease is of importance to reduce the incidence of ischemic complications. On the other hand, shock on admission, which in trauma patients is mainly caused by haemorrhage, does not seem to increase the risk for MI in our study.

Coagulopathy in bleeding trauma patients is often associated with disseminated intravascular coagulation (DIC). Accordingly, we assessed the impact of trauma coagulopathy on the development of TE events. Our analysis showed that coagulopathy doesn't influence the risk for either venous or arterial TE events. Moreover, use of coagulation factors was an independent risk factor for the development of TE events. Nevertheless, we weren't able to identify the individualized risk factors for each factor due to the small number size. Several studies have demonstrated that FVIIa might represent a risk factor in certain patients groups [24], while others weren't able to find evidence in applying fibrinogen concentrate or rFVIIa [25] associated with a higher risk for thrombotic complications.

\section{Limitations}

We recognize several limitations in our study. Although there is an internet-based integrated plausibility check, data quality and completeness of the documented parameters tend to be lower in registries as compared to prospective clinical studies. Comorbidities as well as medication were not documented in the TraumaRegister $\mathrm{DGU}^{\circledR}$. The documentation of TEs was largely limited to the level of "present / not present". Some details that could prove these complications (like mode of detection) were collected in a limited amount from 2005-2009 only. Thus the rate of TE reported here is based on clinically relevant events documented in the patients' files, and not on a systematically and equally applied diagnostic process. Moreover, our study design is only able to show associations; the proof of causal relationships needs other methodological approaches.

\section{Conclusion}

In conclusion, the present study confirms that trauma patients have an altered clinical outcome if they suffer from thromboembolic complications. The most important risk factors are age $>60$ and multiple surgeries.

Conflict of interest All authors declare no conflict of interests.

\section{References}

1. Guryel E, Pearce R, Rickman M, Bircher M (2012) Thromboprophylaxis in pelvic and acetabular trauma patients: a UK consensus? Int Orthop 36(1):165-169

2. Geerts WH, Pineo GF, Heit JA, Bergqvist D, Lassen MR, Colwell CW, Ray JG (2004) Prevention of venous thromboembolism: the Seventh ACCP Conference on Antithrombotic and Thrombolytic Therapy. Chest 126(3 Suppl):338S-400S

3. Paffrath T, Wafaisade A, Lefering R, Simanski C, Bouillon B, Spanholtz T, Wutzler S, Maegele M, Trauma Registry of DGU (2010) Venous thromboembolism after severe trauma: incidence, risk factors and outcome. Injury 41(1):97-101

4. Di Minno MN, Tufano A, Ageno W, Prandoni P, Di Minno G (2012) Identifying high-risk individuals for cardiovascular disease: similarities between venous and arterial thrombosis in perspective. A 2011 update. Intern Emerg Med 7(1):9-13

5. Burke JF, Stulc JL, Skolarus LE, Sears ED, Zahuranec DB, Morgenstern LB (2013) Traumatic brain injury may be an independent risk factor for stroke. Neurology 81(1):33-39

6. Kamel H, Johnston SC, Kirkham JC, Turner CG, Kizer JR, Devereux RB, Iadecola C (2012) Association between major perioperative hemorrhage and stroke or Q-wave myocardial infarction. Circulation 126(2):207-212

7. Aldemir M, Tacyildiz I, Girgin S (2004) Predicting factors for mortality in the penetrating abdominal trauma. Acta Chir Belg 104(4): 429-434

8. Cohen AT, Tapson VF, Bergmann JF, Goldhaber SZ, Kakkar AK, Deslandes B, Huang W, Zayaruzny M, Emery L, Anderson FA Jr et al (2008) Venous thromboembolism risk and prophylaxis in the acute hospital care setting (ENDORSE study): a multinational crosssectional study. Lancet 371(9610):387-394

9. Yassin M, Mitchell C, Diab M, Senior C (2014) The necessity of pharmacological prophylaxis against venous thromboembolism in major joint arthroplasty. Int Orthop 38(5):1073-1075

10. Franchini M, Mannucci PM (2012) Association between venous and arterial thrombosis: clinical implications. Eur J Intern Med 23(4): 333-337

11. Brenneman FD, Redelmeier DA, Boulanger BR, McLellan BA, Culhane JP (1997) Long-term outcomes in blunt trauma: who goes back to work? J Trauma 42(5):778-781

12. Adams SD, Cotton BA, McGuire MF, Dipasupil E, Podbielski JM, Zaharia A, Ware DN, Gill BS, Albarado R, Kozar RA et al (2012) Unique pattern of complications in elderly trauma patients at a level I trauma center. J Trauma Acute Care Surg 72(1):112118

13. Stawicki SP, Grossman MD, Cipolla J, Hoff WS, Hoey BA, Wainwright G, Reed JF 3rd (2005) Deep venous thrombosis and pulmonary embolism in trauma patients: an overstatement of the problem? Am Surg 71(5):387-391 
14. Montgomery KD, Geerts WH, Potter HG, Helfet DL (1996) Thromboembolic complications in patients with pelvic trauma. Clin Orthop Relat Res 329:68-87

15. Tawil I, Stein DM, Mirvis SE, Scalea TM (2008) Posttraumatic cerebral infarction: incidence, outcome, and risk factors. J Trauma 64(4):849-853

16. Assmann G, Cullen P, Schulte H (2002) Simple scoring scheme for calculating the risk of acute coronary events based on the 10-year follow-up of the prospective cardiovascular Munster (PROCAM) study. Circulation 105(3):310-315

17. Britt LD, Zolfaghari D, Kennedy E, Pagel KJ, Minghini A (1996) Incidence and prophylaxis of deep vein thrombosis in a high risk trauma population. Am J Surg 172(1):13-14

18. Anderson FA Jr, Spencer FA (2003) Risk factors for venous thromboembolism. Circulation 107(23 Suppl 1):I9-16

19. Gearhart MM, Luchette FA, Proctor MC, Lutomski DM, Witsken C, James L, Davis K Jr, Johannigman JA, Hurst JM, Frame SB (2000) The risk assessment profile score identifies trauma patients at risk for deep vein thrombosis. Surgery 128(4):631-640
20. Cushman M (2007) Epidemiology and risk factors for venous thrombosis. Semin Hematol 44(2):62-69

21. Knudson MM, Ikossi DG (2004) Venous thromboembolism after trauma. Curr Opin Crit Care 10(6):539-548

22. Toker S, Hak DJ, Morgan SJ (2011) Deep vein thrombosis prophylaxis in trauma patients. Thrombosis 2011:505373

23. Greenfield LJ, Proctor MC, Rodriguez JL, Luchette FA, Cipolle MD, Cho J (1997) Posttrauma thromboembolism prophylaxis. J Trauma 42(1):100-103

24. Lau P, Ong V, Tan WT, Koh PL, Hartman M (2012) Use of activated recombinant factor VII in severe bleeding - evidence for efficacy and safety in trauma, postpartum hemorrhage, cardiac surgery, and gastrointestinal bleeding. Transf Med Hemother 39(2):139-150

25. Chen H, Xue LX, Guo Y, Chen SW, Wang G, Cao HL, Chen J, Tian HL (2013) The influence of hemocoagulation disorders on the development of posttraumatic cerebral infarction and outcome in patients with moderate or severe head trauma. BioMed Res Int 2013:685174 